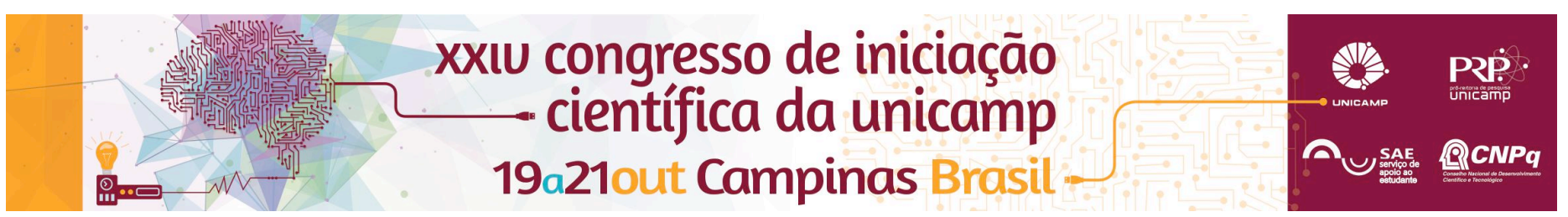

\title{
Identification and structuring of startups in the IT industry
}

\section{Arthur M. Mendes*, Eduardo G. Amaral}

\begin{abstract}
In the present study, it was identified the main regions in Brazil with the biggest development and the biggest potential of growth in the IT industry alongside with their main structures of support. The organizational structures of the main startups in these regions were also studied.
\end{abstract}

\section{Key words: \\ Startup, Accelerator, IT}

\section{Introduction}

The IT industry in the last decades has been a factor of acceleration in the growth of the main global economies. What can be perceived with the analysis of some aspects that accompany its developement and maturation is the generation of jobs, the general growth of productivity, the rise in the indexes of competitivity and in the GDP per capita.

According to data from 2011, Brazil has the 7th biggest internal market on Information Technology and Communication (ITC) what makes for $8 \%$ of its GDP.

Together with an internal market with excellent conditions, the startup environment is being developed all over the country and has shown an exponencial growth is the previous years. Many different types of support structures are already a reality in Brazil like angel investors, accelerators and incubators.

In this project, the main objectives were to:

1 Identify the Brazilian regions with the biggest development and the biggest potential of growth in the IT industry

2 Identify the main structures of support in these regions

3 Study the organizational structures of the main startups of these regions

\section{Results and Discussion}

To achieve objective 1 , the 2012 report from the SOFTEX observatory was used alongside with the website www.startupbase.net.

To achive objective 2, the database from the website Startupi and an article from UOL Economia were taken as reference.

To achieve objective 3, a criterious research was made through the internet to define the startups to study and plenty of benchmarkings were realized in order to better know their organizational structure.

The following data was compiled from the 2012 report from the SOFTEX observatory:

\section{Net revenue generated by IT companies per state}

$\mathrm{R} \$ 29.577 .535-64,2 \%$ - SP

$\mathrm{R} \$ 09.710 .158-21,1 \%-\mathrm{RJ}$

$\mathrm{R} \$ 02.326 .426-05,1 \%-\mathrm{MG}$

$\mathrm{R} \$ 01.732 .113-03,8 \%$ - RS

$\mathrm{R} \$ 01.487 .303-03,2 \%$ - SC

$\mathrm{R} \$ 01.225 .557-02,7 \%-\mathrm{PR}$

Table 1. Number of startups, angel inverstors and accelerators per state

\begin{tabular}{|c|c|c|c|}
\hline & Startup & Angel Investors & Accelerators \\
\hline RJ & 192 & 62 & 5 \\
\hline SP & 696 & 47 & 14 \\
\hline MG & 210 & 10 & 5 \\
\hline PR & 109 & 11 & 3 \\
\hline PE & 88 & 8 & 1 \\
\hline
\end{tabular}

\section{Benchmarks}

WeDoLogos: "We are divided per areas: Customer service, Sales, Marketing and IT."

RunRun.it: "We have 3 big nuclei: IT, Marketing and Customer Success."

EadBox: "We have a dynamic structure with areas of Marketin, Projects, Development, Support, Commercial, Financial and Legal. But the employees can be in more than one area when needed."

\section{Conclusions}

The results lead us to conclude that Rio de Janeiro and Sao Paulo are the two regions in Brazil with the biggest development in the IT industry and also with the biggest potential of growth. As for the organizational structure of the startups, they don't follow any particular pattern and are mostly driven by the skills and needs of the entrepreneurs.

\section{Number of IT companies per region}

$30.188-54.3 \%$ - SP

$07.952-14,3 \%$ - RJ

$05.513-09,9 \%-$ RS

$05.006-09,0 \%-$ MG

$04.447-08,0 \%-P R$

$02.470-04,4 \%-$ SC 